\title{
A User-Centred Approach to Reducing Sedentary Behaviour
}

\author{
Chelsea Dobbins, Madjid Merabti, Paul Fergus, David Llewellyn-Jones \\ School of Computing and Mathematical Sciences \\ Liverpool John Moores University \\ Liverpool, UK \\ \{C.M.Dobbins, M.Merabti, P.Fergus, D.Llewellyn-Jones\} @1jmu.ac.uk
}

\begin{abstract}
The use of digital technologies in the administration of healthcare is growing at a rapid rate. However, such platforms are often expensive. As people are living longer, the strain placed on hospitals is increasing. It is evident that a usercentric approach is needed, which aims to prevent illness before a hospital visit is required. As such, with the levels of obesity rising, preventing this illness before such resources are required has the potential to save an enormous amount of time and money, whilst promoting a healthier lifestyle. New and novel approaches are needed, which are inexpensive and pervasive in nature. One such approach is to use human digital memories. This outlet provides visual lifelogs, composed of a variety of data, which can be used to identify periods of inactivity. This paper explores how the DigMem system is used to successfully recognise activity and create temporal memory boxes of human experiences, which can be used to monitor sedentary behaviour.
\end{abstract}

Index Terms-Human Digital Memory, Lifelogging, Sensors, Physiological Monitoring, Pervasive Healthcare

\section{INTRODUCTION}

The deliverance of healthcare is going through a major transition. Before the $20^{\text {th }}$ century, medical care was given at home, with visits from family physicians. With the advancement in technology, rare and expensive resources, such as heavy technology and specialist equipment, had to be centralized in hospitals to make utilization effective [1]. However, with the life expectancy of adults in Great Britain increasing [2], so is the occurrence of illness, disability and the demand on hospitals. The strain placed on these resources is evident, and clearly, a user-centric approach is needed, which aims to prevent illness before a hospital visit is required.

eHealth, a term often used to describe the use of digital technologies in the administration and communication of healthcare, is growing at a rapid rate [3]. England, for example, has invested at least $£ 12.8$ billion into a National Programme for Information Technology (NPfIT), for the National Health Service (NHS) [3]. Similarly, the United States (US) have committed a $\$ 38$ billion eHealth investment into health care [3]. The phrase "eHealth" encompasses a variety of disciplines, including Pervasive Healthcare, Mobile Healthcare (mHealth) and Personal Healthcare (pHealth), to name but a few. Specifically, pervasive healthcare can be defined from two perspectives. Firstly, as the application of pervasive computing, or ubiquitous computing for healthcare; second, as making healthcare available everywhere, anytime, pervasively [4]. One of the most important application areas for pervasive healthcare is the support that it provides for independent living, wellness and disease management [4].

Influencing behavioural health outcomes, using eHealth platforms, has great promise. This is due to its enhanced features of interactivity, multimodality, mass customization, and the opportunity for users to also be producers [5]. In particular, reducing sedentary behaviour, utilizing these technologies has enormous potential. Sedentary behaviour is a class of behaviours that involves low levels of energy expenditure and are associated with an increased risk of obesity and cardiovascular disease [6]. The effect of the rising levels of obesity is evident throughout the NHS's Statistics on Obesity, Physical Activity and Diet report [6]. According to their statistics, "In England, in 2002, physical inactivity was estimated to cost at least $£ 2$ billion and maybe up to £8.2 billion a year and does not include the contribution of physical inactivity to obesity estimated at $£ 2.5$ billion annually". With these figures, combating sedentary behaviour is essential, not only for the benefit of the health services but also for our own health and wellbeing. In terms of influencing behaviour, the use of eHealth, interpersonal, approaches are effective in changing individual behaviour. However, they are often too expensive and limited in reach to have a population effect [5]. New and novel approaches are needed, which are inexpensive and ubiquitous in nature.

Addressing these issues, via Human Digital Memories (HDM), is a viable option. HDMs are vast digital archives of one's personal life experiences and are constructed from a wide range of data sources, across various media types [7], [8]. For example, content recorded from someone's life might include all photographs taken; videos seen; music listened to; details of places visited; physiological signals; details of people met etc. [8]. Utilizing ordinary devices, embedded with sensors, a variety of data, about ourselves and surroundings, can be captured. Bringing all of these disjointed pieces of information together enables selfreflection to occur, and we can see how our actions influence our health. HDMs provide a visual illustration of our activities and the state that our bodies were in, at any one time. In order to prevent the onset of obesity, this depiction of how our bodies have changed over the years would be the incentive that people would need to change their lifestyles. However, searching and processing this data and classifying behaviour, from this disjointed set of information, is a challenge. A new and novel platform is needed, which 
addresses this and provides a way to bring together and extract relevant information from information accumulated over a lifetime. In realizing this, the DigMem system [9]-[12] utilizes distributed mobile services, linked data and machine learning to create rich and interactive human digital memories. In this way, information is structured to create temporal memory boxes of human experiences. The system is also able to answer life questions about our human digital memory data. Using this system [9]-[12], the paper aims to address these challenges and explores how activity can be recognised, in order to create memory boxes, which can be used to monitor sedentary behaviour.

\section{BACKGROUND}

Research into capturing and creating HDMs has received a great deal of attention, from researchers, over the last few decades. Since the Memex [13] in 1945, research into how aspects of our lives can be captured and organised, have been investigated. Over time, this vision of storing accumulated items has evolved into digitally capturing information about ourselves and our environment. The culmination of this practise has been to continually capture content, with the aid of wearable systems (lifelogging).

Microsoft's SenseCam [14] is a revolutionary lifelogging device, which is capable of storing up to 30,000 images [14]. The device contains a digital camera, with a fisheye lens, and multiple sensors. On-board there are sensors to detect changes in light levels, an accelerometer, a thermometer, and a passive infrared sensor to detect the presence of people [14]. Originally developed as a retrospective memory aid, its application within behavioural studies, is also gaining momentum. These investigations are opening up a whole new way in which HDMs can impact our physical health.

One such approach has been Lindley et al.'s [15] study on creating 'small stories' based around the SenseCam images. By reflecting upon the images and discussing and re-creating memories, associated with the photos, these helped the users to reflect upon daily life and to identify periods of sedentary behaviour. This study was supported by Doherty et al. [16] who stated that "After participants looked at their images, they were prompted to change their lifestyle by, for example, cycling instead of driving, taking up exercise, and spending more time interacting with their children". This study also emphasizes the importance that visual illustrations of behaviour play on changing our habits and has been particularly useful for developing automatic classifiers for graphical life-logs to infer different lifestyle traits or characteristics [16]. It has also been beneficial because of the amount of data that was collected and used. The study included 95,000 manually annotated images and 3 million lifelog images, obtained from 33 individuals, sporadically, over a period of 3.5 years. These images were later used to identify 22 different lifestyle traits, which were then analysed to inform individuals about their future wellbeing [16].

Mobile devices and sensor equipment are also able to capture a more comprehensive record of everyday life, as continuously as possible [17]. As people become more interested in monitoring their health, physiological devices are becoming smaller and more practical to wear on a daily basis. These devices can record a range of information about ourselves, and coupled with other collected data items, can offer a way to see how we were feeling at any point in our lives. Integrating sensors into everyday clothing, using "smart fabrics and interactive textiles (SFIT)" [18], is one such practical solution. Sensors don't have to be placed on the body by a professional; therefore, the user can be monitored at any time [18]. One such approach, in this area, has been López et al.'s [19] LOBIN project. A combination of e-textile and wireless sensor networks have been used to provide an efficient way to support non-invasive and pervasive services [19]. The system consists of a set of "smart shirts" that monitor electrocardiogram (ECG) heart rate, angle of inclination, activity index, and body temperature and a location subsystem, which monitors the patient's location [19]. The information is then sent to the management subsystem, which processes and stores the data. This system is quite interesting due to the parameters that can be measured and the location system, particularly as patients are tracked indoors.

As it can be seen, there are many approaches that are used to capture data. However, these methods are used in very separate fields and have rarely been combined. In order to form a more rounded snapshot of our lives these technologies need to work together. So that not only can a visual representation of experiences be recapped, but also the feelings and changes our bodies were experiencing when those events were occurring. Subsequently, by incorporating even more data, for instance, from smart objects and our environment, would reduce ambiguity further. This information could also be used to establish how different environments affect us. For example, a higher heart rate, than normal, and an increase in sweat production could be attributed to many things. Presenting only this information, as a memory, is insufficient. However, if it was known that it was a hot day, by incorporating a temperature reading and that a photo of the user doing physical activity was also obtained, then the context of the physiological data is known. Bringing together data, from separate sources, enables a finer level of detail to be achieved, as the range of accessible information is increased. In order to integrate this data into HDMs, advanced solutions are needed. A significant drawback is the ambiguity of physiological data, which can require extensive data analysis. Automatic analysis of this data would have to be performed in order to discern meaningful information to enhance our memories.

\section{ThE DIgMEM SySTEM}

In previous work [20], the key research challenges of utilizing HDM's for the purpose of preventing sedentary behaviour have been discussed. One such challenge, which has been identified, was the classification of behaviour. Body sensors provide streams of data, which allow specific episodic manifestations to be monitored, e.g. sitting, walking or running. However, mapping these isolated data streams, and their relationships with others, to infer behaviour was a significant challenge.

In realising this challenge, current research focuses on classifying behaviour, in order to answer life questions about the user's data. Based on this, temporal memory boxes of human experiences are created [10]. This approach enables the searching of HDM data to be treated as a machine learning problem, which eliminates the need to understand a 
complicated language, e.g. SPARQL [21]. SPARQL is a very complex language, and if the queries are not constructed precisely then false results can occur. However, by using a probabilistic approach, an exact query match is not required. This approach enables specific questions to be answered about ourselves, such as "Have I ran before?" "Does this location make me happy?" or "Do I run enough?" The ability to answer such abstract questions is a unique feature that has not been seen before.

In order to demonstrate this idea, a sample of location, heart rate and data from 3 tri-axil accelerometers, located on the ankle, chest and hand have been used. Various activities have been undertaken to gather a diverse range of data. The activities that have been chosen are lying down, sitting, standing, walking, running, ascending/descending stairs, vacuum cleaning and ironing. Each activity has been performed for approximately 3 minutes. These behaviours have been chosen because they are a good mixture of high and low energy actions.

In demonstrating this idea, the question, "Have I sat down today?" has been answered, using the decision tree (TREEC) classifier. This algorithm has been chosen because, during initial tests, it has produced good results [11].Table 1 illustrates the resulting confusion matrix from this test. As it can be seen, the classifier has been $100 \%$ successful in recognizing sitting. Overall, the majority of activities have been correctly identified, with only four errors occurring. In this instance, the system has been able to recognise that the user has sat down and thus has successfully answered the question. As the system learns more about the user, more probing questions will begin to emerge. For instance, emotional features can be used to determine if certain locations make us happy. This has profound implications and provokes a deeper understanding and reflection of ourselves. This aspect of the system demonstrates that activities can be recognised but not the context of those times. In this instance, a memory box is used to illustrate the user's interactions, movements and physiological signals and to visualise any time of our lives.

Figure 1 demonstrates this idea. Each input (Location, Photos, Acceleration and Heart Beat), and examples of potential other input devices that can provide information, are displayed in the memory box. When an input is clicked on, a separate window opens, and a more in-depth illustration of the data is seen. Figure 2 a) illustrates the user's location, whilst Figure 2 b) displays any collected photos. Figure 2 c) and d) illustrates the user's acceleration and heart rate data.

\begin{tabular}{|c|c|c|c|c|c|c|c|c|c|}
\hline \multirow{2}{*}{ True Labels } & \multicolumn{9}{|c|}{ Estimated Labels } \\
\cline { 2 - 9 } & Lown & Sitting & Standing & Walking & Running & $\begin{array}{c}\text { Walking } \\
\text { Up } \\
\text { Stairs }\end{array}$ & $\begin{array}{c}\text { Walking } \\
\text { Down } \\
\text { Stairs }\end{array}$ & Vacuuming & Ironing \\
\hline Lying Down & 11 & & & & & & & & \\
\hline Sitting & & 11 & & & & & & & \\
\hline Standing & & & 10 & & & 1 & & & \\
\hline Walking & & & & 11 & & & & & \\
\hline Running & & & & & 11 & & & & \\
\hline $\begin{array}{c}\text { Walking Up } \\
\text { Stairs }\end{array}$ & & & & & & 10 & & & 1 \\
\hline $\begin{array}{c}\text { Walking } \\
\text { Down Stairs }\end{array}$ & & & & & & & 11 & & \\
\hline Vacuuming & & & & 1 & & & & 10 & \\
\hline Ironing & & & & & & & 1 & & 10 \\
\hline
\end{tabular}

Table 1 Confusion Matrix Results



Figure 1 DigMem Memory Box

Drilling deeper into this memory box, by utilizing accelerometer and heart rate information, activities begin to emerge. Using all of this information it is possible to understand and see exactly what our bodies were going through at the time. In this instance, the user was sitting at their desk, at Liverpool John Moores University, which has been validated by the memory box data (see Figure 2).

Obtaining and linking data from a variety of sources provides a greater level of detail in the creation of human digital memories. This test has successfully demonstrated how disjointed pieces of information can be brought together to form a memory box of a particular time. These boxes can pinpoint activities and help us to relive any moment of our life. As well as this aspect, memory boxes also help us to understand and see how our behaviours have changed over time. By providing, an outlet that highlights the amount of time spent in periods of inactivity is the evidence that is needed to change behaviour. 


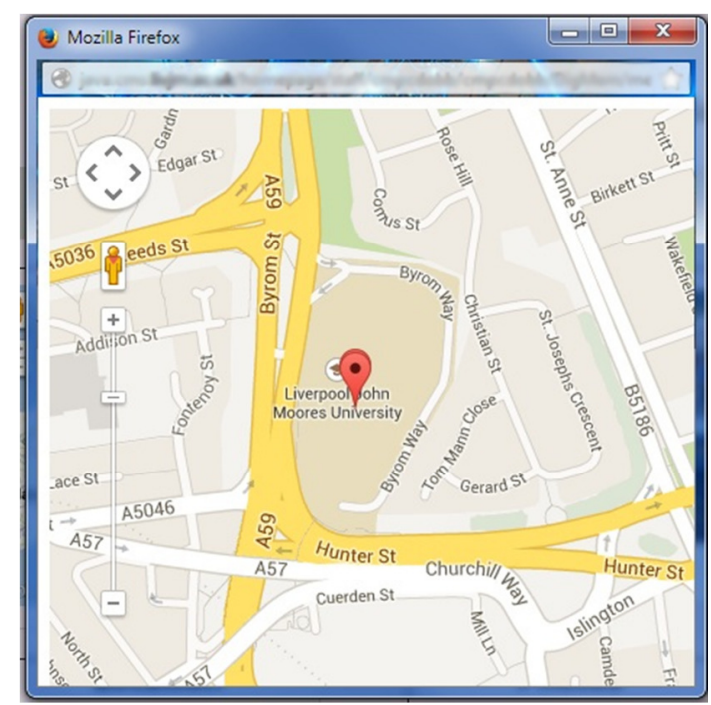

a) Location



c) Acceleration



b) Photos



d) Heart Rate

Figure 2 DigMem Memory Box Data

\section{Discussion}

In this scenario, the system identified sitting and presented this activity as a memory box. DigMem offers a new way to, as unobtrusively as possible, monitor a user, for potentially their entire lives. By providing visual evidence that a user has been spending too much time in sedentary positions it is hoped that this is all that they will need to change their behaviour. The system is also able to provide information to individuals about themselves and their behaviour patterns. This information can then be used to implement compensatory changes and view the impact this has on specific medical outcomes.

Obtaining and linking data from a variety of sources provides a greater level of detail in the creation of human digital memories. Adding as much detail as possible enables the execution of smart queries, which have the ability to search data in a multi-dimensional fashion. For example, in a home scenario, sensors attached to a user's body are able to record any movements and physiological data, whilst static sensors, attached to the sofa, monitor the pressure that is being exerted, in particular, pressure point hotspots. A camera, hanging from their neck, is capturing photos every $x$ minutes and there are sensors in the room that are monitoring the state of the room, i.e. temperature, humidity, etc. Data from a smart TV is gathering viewing times, whilst sensors in the fridge and cupboards monitor food intake. Their mobile device is continually broadcasting messages for the automatic retrieval of data from these devices, prevalent within their environment. Using DigMem, the collected data is then transformed and added to the HDM feature space [9]. By searching this space, extended periods of inactivity can be identified and memory boxes can be created from the data.

Memory boxes provide the visual evidence that is needed in determining if a user has been sedentary for too long and if their diet is particularly poor. Any time, throughout our lives, 
can be reconstructed and our feelings, from those times, reasoned over. This is a very powerful feature of the system, which makes it unique. Human memories are infinite, and their digital counterparts should not be any different. The system can track our entire lives and monitor a user for any amount of time; something that current systems are unable to do.

\section{SUMMARY AND FUTURE WORK}

Enabling users to take a more active role in preventing the onset of certain diseases has the potential to alleviate the strain that is placed on hospitals and care staff. As users become more interested in monitoring their behaviour and sensors become more prevalent, within the environment, a great deal of information can be collected. Moreover, the advancement in physiological devices enables users to track themselves and to monitor their health and wellbeing, with minimal effort. However, with all of this data available, there are limited channels that are able to harvest this information and transform it into meaningful material. The DigMem system addresses this limitation by enabling life questions to be answered and fragmented data to be transformed into temporal memory boxes of human experiences. In this sense, the user is able to see exactly where they were, what they were doing and how their bodies were reacting. As demonstrated in this work, life questions can also be asked about this data, such as "Have I been sitting today?" "Does this location make me happy?" or "Have I run before?" This enables us to gain a deeper insight into our behaviours and to isolate these times, to see exactly what was happening.

Future work aims to build on these user questions with the development of emotional classification algorithms. Recent research into the area of affective computing has demonstrated that emotional states of people can be recognized from their physiological signals [22]-[24]. Currently, the system utilizes this type of information, in the form of heartbeat and acceleration signals, and links this data with photographic and location information to infer emotions from events. Whilst this is a good starting point, further examination, into this point, is required so that the system knows the features of being happy, sad, angry, etc. so that data can be classified in this way. Furthermore, executing queries that require sophisticated interpretation is another interesting point, such as 'find a picture of me playing with Peter when he was a toddler' [25]. This type of query places considerable focus on computer vision and image understanding [26], [27]. In order to execute this query, an innate understanding of who the people in the picture are and activity recognition are required. These points are very interesting and will be propelling the research, into this, area forward.

\section{REFERENCES}

[1] C. E. Koop, R. Mosher, L. Kun, J. Geiling, E. Grigg, S. Long, C. Macedonia, R. C. Merrell, R. Satava, and J. M. Rosen, "Future Delivery of Health Care: Cybercare," IEEE Eng. Med. Biol. Mag., vol. 27, no. 6, pp. 29-38, Jan. 2008.

[2] Office for National Statistics, "Healthy Life Expectancy: Living Longer In Poor Health,” 2010. [Online]. Available: http://www.statistics.gov.uk/cci/nugget.asp?id=2159. [Accessed: 01-Jun-2013].

[3] A. D. Black, J. Car, C. Pagliari, C. Anandan, K. Cresswell, T. Bokun, B. McKinstry, R. Procter, A. Majeed, and A.
Sheikh, "The Impact of eHealth on the Quality and Safety of Health Care: A Systematic Overview," PLoS Med., vol. 8, no. 1, pp. 1-16, Jan. 2011.

[4] I. Korhonen and J. E. Bardram, "Guest Editorial Introduction to the Special Section on Pervasive Healthcare," IEEE Trans. Inf. Technol. Biomed., vol. 8, no. 3, pp. 229-234, Sep. 2004.

[5] L. Neuhauser and G. L. Kreps, "eHealth communication and behavior change: promise and performance," Soc. Semiot., vol. 20, no. 1, pp. 9-27, Feb. 2010.

[6] The NHS Information Centre Lifestyles Statistics, "Statistics on Obesity, Physical Activity and Diet: England, 2012," 2012.

[7] C. Gurrin, D. Byrne, N. O'Connor, G. J. F. Jones, and A. F. Smeaton, "Architecture and Challenges of Maintaining a Large-scale, Context-aware Human Digital Memory," in 5th International Conference on Visual Information Engineering, 2008, pp. 158-163.

[8] L. Kelly and G. J. F. Jones, "Venturing into the labyrinth: the information retrieval challenge of human digital memories," in Workshop on Supporting Human Memory with Interactive Systems, Lancaster, UK, 2007, pp. 37-40.

[9] C. Dobbins, M. Merabti, P. Fergus, D. Llewellyn-Jones, and F. Bouhafs, "Exploiting linked data to create rich human digital memories," Comput. Commun. (In Press., vol. 36, no. 15-16, pp. 1639-1656, Jul. 2013.

[10] C. Dobbins, M. Merabti, P. Fergus, and D. LlewellynJones, "Creating Human Digital Memories for a Richer Recall of Life Experiences," in Proceedings of 10th IEEE International Conference on Networking, Sensing and Control (ICNSC'13), 2013, pp. 246-251.

[11] C. Dobbins, M. Merabti, P. Fergus, and D. LlewellynJones, "Creating Human Digital Memories with the Aid of Pervasive Mobile Devices," Pervasive Mob. Comput. J., 2013.

[12] C. Dobbins, M. Merabti, P. Fergus, and D. LlewellynJones, "Capturing and Sharing Human Digital Memories with the Aid of Ubiquitous Peer- to-Peer Mobile Services," in Proceedings of 10th Annual IEEE International Conference on Pervasive Computing and Communications (PerCom'12), 2012, pp. 64-69.

[13] V. Bush, "As We May Think," The Atlantic Monthly, no. JULY 1945, 1945.

[14] S. Hodges, L. Williams, E. Berry, S. Izadi, J. Srinivasan, A. Butler, G. Smyth, N. Kapur, and K. Wood, "SenseCam: A Retrospective Memory Aid," UbiComp 2006 Ubiquitous Comput., vol. 4206, pp. 177-193, 2006.

[15] S. E. Lindley, D. Randall, W. Sharrock, M. Glancy, N. Smyth, and R. Harper, "Narrative, Memory and Practice: Tensions and Choices in the Use of a Digital Artefact," in Proceedings of the 23rd British HCI Group Annual Conference on People and Computers: Celebrating People and Technology, 2009, pp. 1-9.

[16] A. R. Doherty, N. Caprani, C. Ó. Conaire, V. Kalnikaite, C. Gurrin, A. F. Smeaton, and N. E. O'Connor, "Passively Recognising Human Activities Through Lifelogging," Comput. Human Behav., vol. 27, no. 5, pp. 1948-1958, Sep. 2011.

[17] A. J. Sellen and S. Whittaker, "Beyond Total Capture: A Constructive Critique of Lifelogging," Commun. ACM, vol. 53, no. 5, pp. 70-77, May 2010.

[18] A. Lymberis and R. Paradiso, "Smart Fabrics and Interactive Textile Enabling Wearable Personal Applications: R\&D State of the Art and Future Challenges," in 30th Annual International Conference of the IEEE Engineering in Medicine and Biology Society, 2008, vol. 2008, pp. 5270-5273.

[19] G. López, V. Custodio, and J. I. Moreno, "LOBIN: Etextile and wireless-sensor-network-based platform for 
healthcare monitoring in future hospital environments," IEEE Trans. Inf. Technol. Biomed., vol. 14, no. 6, pp. 1446-1458, Nov. 2010.

[20] C. Dobbins, P. Fergus, M. Merabti, and D. LlewellynJones, "Monitoring and Measuring Sedentary Behaviour with the Aid of Human Digital Memories," in Proceedings of The 9th Annual IEEE Consumer Communications \& Networking Conference (CCNC'12), 2012, pp. 395-398.

[21] W3C, "SPARQL Query Language for RDF," 2008. [Online]. Available: http://www.w3.org/TR/rdf-sparqlquery/. [Accessed: 10-Aug-2013].

[22] L. Ivonin, H.-M. Chang, W. Chen, and M. Rauterberg, "Unconscious emotions: quantifying and logging something we are not aware of," Pers. Ubiquitous Comput., vol. 17, no. 4, pp. 663-673, Apr. 2012.

[23] E. L. van den Broek, J. H. Janssen, and J. H. D. M. Westerink, "Guidelines for Affective Signal Processing (ASP): from lab to life," in 3rd International Conference on Affective Computing \& Intelligent Interaction, ACII 2009, 10-12 Sept 2009, Amsterdam, The Netherlands., 2009.
[24] D. McDuff, A. Karlson, A. Kapoor, A. Roseway, and M. Czerwinski, "AffectAura: An Intelligent System for Emotional Memory," in Proceedings of the 2012 ACM annual conference on Human Factors in Computing Systems - CHI '12, 2012, pp. 849-858.

[25] A. Fitzgibbon and E. Reiter, "Grand Challenges in Computing Research: GC3 Memories for life: managing information over a human lifetime," in Conference on Grand Challenges in Computing Research, 2005, pp. 1316.

[26] V. Delaitre, I. Laptev, and J. Sivic, "Recognizing human actions in still images: a study of bag-of-features and partbased representations," Procedings Br. Mach. Vis. Conf., vol. 2, no. 5, p. 7, 2010.

[27] N. Bicocchi, M. Lasagni, and F. Zambonelli, "Bridging Vision and commonsense for Multimodal Situation Recognition in Pervasive Systems," in IEEE International Conference on Pervasive Computing and Communications (PerCom), 2012, pp. 48-56. 